\title{
REGULAR ELEMENTS DETERMINED BY GENERALIZED INVERSES
}

\author{
ADEL ALAHMADI, S. K. JAIN, AND ANDRÉ LEROY
}

\begin{abstract}
In a semiprime ring, von Neumann regular elements are determined by their inner inverses. In particular, for elements $a, b$ of a von Neumann regular ring $R, a=b$ if and only if $I(a)=I(b)$, where $I(x)$ denotes the set of inner inverses of $x \in R$. We also prove that, in a semiprime ring, the same is true for reflexive inverses.
\end{abstract}

\section{INTRODUCTION AND PRELIMINARIES}

In this short note, $R$ will stand for an associative ring with unity. An element $a \in R$ is (von Neumann) regular if there exists $x \in R$ such that $a=a x a$. Such an element $x$ is called an inner inverse (also called von Neumann inverse or generalized inverse) of $a$. The set of regular elements of a ring $R$ is denoted by $\operatorname{Reg}(R)$. A ring $R$ is regular if $\operatorname{Reg}(R)=R$. Note that a regular ring is semiprime. In general, a regular element may have more than one inner inverse. We denote the set of inner inverses of $a$ by $I(a)$. An element $x \in R$ is called an outer inverse of $a$ if $x a x=x$. Note that if $x \in I(a)$ then $x a x$ is both an inner and an outer inverse of $a$. An element $x \in R$ is called a reflexive inverse of $a$ if it is both an inner and an outer inverse of $a$. Denote the set of reflexive inverses of $a$ by $\operatorname{Re} f(a)$. We first obtain a necessary and sufficient condition for $I(a) \subseteq I(b)$ (Lemma 6) and use this to prove that in a semiprime ring, for $a, b \in \operatorname{Reg}(R), I(a)=I(b)$ if and only if $a=b$ if and only if $\operatorname{Ref}(a)=\operatorname{Ref}(b)$. (Theorem[7 and Theorem[10).

We begin with a few key lemmas.

The following is well-known (cf. [1] Corollary 1, Chapter 2. p. 40.)

Lemma 1. For $a \in R$ and $a_{0} \in I(a)$, we have $I(a)=\left\{a_{0}+t-a_{0} a t a a_{0} \mid t \in R\right\}$.

As usual, $l(a)$ and $r(a)$ denote respectively the left and right annihilator of an element $a \in R$. We define the inner annihilator of an element $a \in R$, as $\{x \in R \mid a x a=0\}$ and denote it by $\operatorname{Iann}(a)$.

The next Proposition gives a link between $I(a)$ and $\operatorname{Ref}(a)$.

Proposition 2. For $a \in \operatorname{Reg}(R)$, let $\varphi_{a}: I(a) \longrightarrow \operatorname{Ref}(a)$ be such that $\varphi_{a}(x)=$ xax. Then

(1) The map $\varphi_{a}$ is onto.

(2) $\operatorname{Ref}(a)=I(a) a I(a)$.

(3) If $x, y \in I(a)$ are such that $\varphi_{a}(x)=\varphi_{a}(y)$ then $x-y \in l(a) \cap r(a)$.

(4) Let $x \in \operatorname{Re} f(a)$, then $\varphi_{a}(x)=x$.

Proof. This is clear.

The next lemma is straightforward. 
Lemma 3. Let $a \in \operatorname{Reg}(R)$ and $a_{0} \in I(a)$. Write $e=a a_{0}, f=a_{0} a$ and $e^{\prime}=1-e$, $f^{\prime}=1-f$. Then

(i) $\operatorname{Iann}(a)=l(a)+r(a)=R e^{\prime}+f^{\prime} R$.

(ii) $I(a)=a_{0}+\operatorname{Iann}(a)=a_{0}+R e^{\prime}+f^{\prime} R$.

(iii) If $a_{0} \in \operatorname{Re} f(a)$, then $\operatorname{Ref}(a)=a_{0}+f R e^{\prime}+f^{\prime} R e+f^{\prime} R a R e^{\prime}$.

Proof. We simply mention that the statement (iii) can be proved by using statement (2) of the above proposition 2

\section{Characterization of $I(a)$ and $\operatorname{Re} f(a)$}

We can now state our first result in the following proposition.

Proposition 4. Let $R$ be a semiprime ring. If $a \in R e g(R)$, then for any $b \in R$, $b I(a) b$ is a singleton set if and only if $b \in R a \cap a R$.

Proof. Firstly, suppose that there exist $x, y \in R$ such that $b=x a=a y$ and let $a_{0} \in I(a)$. We then have that, for any $t \in R, b\left(a_{0}+t-a_{0} a t a a_{0}\right) b=\left(x a a_{0}+\right.$ $\left.x a t-x a t a a_{0}\right) a y=x a y+x a t a y-x a t a y=x a y$. This shows that indeed $b I(a) b$ is a singleton set.

Conversely, suppose that $b I(a) b=\left\{b a_{0} b\right\}$. We then have $b\left(a_{0}+t-a_{0} a t a a_{0}\right) b=b a_{0} b$, for any $t \in R$. This implies that, for any $t \in R$, we have

$$
b\left(t-a_{0} a t a a_{0}\right) b=0 .
$$

Substituting $\left(1-a_{0} a\right) t$ for $t$ in this equality leads to $b\left(1-a_{0} a\right) t b=0$, for any $t \in R$. The semiprimeness of $R$ then implies that $b\left(1-a_{0} a\right)=0$, i.e. $b=b a_{0} a \in R a$. Similarly, substituting $t$ by $t\left(1-a a_{0}\right)$ in the above equality gives $b=a a_{0} b$. In particular, $b \in a R$.

We recall the following result obtained by S.K. Jain and M. Prasad ([2]).

Lemma 5. Let $R$ be a ring and let $b, d \in R$ such that $b+d$ is a Von Neumann regular element. Then the following are equivalent:

(1) $b R \oplus d R=(b+d) R$.

(2) $R b \oplus R d=R(b+d)$.

(3) $b R \cap d R=\{0\}$ and $R b \cap R d=\{0\}$.

The next proposition provides necessary and sufficient conditions as to when $I(a) \subseteq I(b)$, where $a, b \in \operatorname{Reg}(R)$ and $R$ is semiprime.

Proposition 6. Let $R$ be a semiprime ring and let $a, b \in \operatorname{Reg}(R)$. Then $I(a) \subseteq I(b)$ if and only if $b R \cap d R=0$ and $R b \cap R d=0$ where $a=d+b$.

Proof. Since $I(a) \subseteq I(b)$, we have $b x b=b$ for every $x \in I(a)$. By Proposition 4. $b \in R a \cap a R$. Write $b=\alpha a=a \beta$ for some $\alpha, \beta \in R$. Then $b I(a) a=b$. Next, $b I(a) d=b I(a) a-b I(a) b=b-b I(a) b=0$. Consider now $d I(a) b=a I(a) b-b I(a) b=$ $a \beta-b I(a) b=b-b=0$. We thus have

$$
b I(a) d=0 \quad \text { and } \quad d I(a) b=0
$$

Then, for any $x \in I(a)$, we have $b+d=a=a x a=(b+d) x(b+d)=b x a+d x b+d x d=$ $b+0+d x d$. This yields,

$$
d I(a) d=d
$$


Now, we proceed to show $d R \cap b R=0$. Let $b x=d y \in b R \cap d R$. Multiplying both sides of the equality (2) by $y$ on the right and using $b x=d y$ we obtain $d I(a) b x=d y$. As proved above, we have $d I(a) b=0$. and so $d y=0$. This proves our assertion. Similarly, we show that $R b \cap R d=0$. Let $x b=y d \in R b \cap R d$. Now, multiplying both sides of the equality (2) on the left by $y$, we get $y d I(a) d=y d$. This proves $x b I(a) d=y d$. Since $b I(a) d=0$, we obtain $y d=0$, proving $R b \cap R d=0$.

The converse is easy using the above lemma 5 .

Next, we show, in particular, that the regular elements of a semiprime ring are equal if their sets of inner inverses are the same.

Theorem 7. Let $R$ be a semiprime ring and $a, b \in \operatorname{Reg}(R)$. Then $I(a)=I(b)$ if and only if $a=b$.

Proof. We only need to prove the sufficency. So assume that $I(a)=I(b)$. Proposotion 6 implies that we can write $a=b+d$ with $b R \cap d R=0, R d \cap R b=0$. Lemma 5 then gives that $(b+d) R=b R \oplus d R$. Since $I(a)=I(b)$ we also have $a I(b) a=\{a\}$ and $b I(a) b=\{b\}$ and Proposition 4 implies that $R a=R b$ and $a R=b R$. This leads to $a R=(b+d) R=b R \oplus d R=a R \oplus d R$. This forces $d$ to be zero and hence $a=b$, as desired.

Alternatively we may invoke Hartwig's result (cf. 3]) in place of Lemma 5 , This was pointed out to us by T.Y. Lam. Indeed, by our Proposition 4 we have $a R=b R$ and $R a=R b$, and thus by invoking Hartwig's result, there exist units $u, v \in R$ such that $b=a u=v a$. If $x \in I(a)=I(b)$, then $a x a=a$ and $b x b=b$. The last equality implies that vaxau $=a u$ and hence $v a=a$. Thus $b=a$.

Corollary 8. Let $R$ be a regular ring. Then $I(a)=I(b)$ if and only if $a=b$.

Remark 9. Pace Nielsen remarked that, in the above theorem, the semiprime hypothesis can be replaced by the assumption that $a-b$ is regular. So assume $I(a)=I(b)$, and $a-b \in \operatorname{Reg}(R)$. As in our Proposition 4 we obtain

$$
b t\left(1-a a_{0}\right) b=0,
$$

for any $t \in R$. If $b_{1}$ is a reflexive inverse of $b$, we obtain, for any $t$ in $R, a t a a_{0} b=$ $\left(a b_{1} a\right) t a a_{0} b=a\left(b_{1} b b_{1}\right) a t a a_{0} b=a b_{1}\left(b b_{1} a t a a_{0} b\right)$. Replace $t$ by $b_{1} a t$ in (1) and obtain

$$
a t a a_{0} b=a t b .
$$

For $z \in I(a-b)$ we have $b z b=b z a+a z b-a z a+a-b$. Using this equality we compute

$$
b z b=b z b a_{0} b=(b z a+a z b-a z a+a-b) a_{0} b=b z a a_{0} b+a z b-a z a a_{0} b+a a_{0} b-b .
$$

Using formulae (1) and (2) we get $a a_{0} b=b$ so that $b R \subseteq a R$. Symmetric arguments leads to $a R=b R$ and $R a=R b$ and Hartwig's theorem finishes the proof. 


\section{REFLEXIVE INVERSES FOR SEMIPRIME RINGS}

We conclude by characterizing the equality of $\operatorname{Re} f(a)=\operatorname{Re} f(b)$, and obtain the analogue of Theorem 7 for reflexive inverses of semiprime rings.

Theorem 10. Let $R$ be a semiprime ring such that $a, b \in \operatorname{Reg}(R)$. Then $\operatorname{Re} f(a)=$ $\operatorname{Re} f(b)$ if and only if $a=b$.

Proof. Let $a_{0} \in \operatorname{Ref}(a)=\operatorname{Ref}(b)$. Since $a=0$ if and only if $\operatorname{Ref}(a)=0$, we may assume that $a$ and $b$ are not zero. Since $b \operatorname{Re} f(a) b=b \operatorname{Re} f(b) b=b$ and $\operatorname{Re} f(a)=I(a) a I(a)$, we have that, for any $t$ in $R$,

$$
b\left(a_{0}+t-a_{0} a t a a_{0}\right) a\left(a_{0}+t-a_{0} a t a a_{0}\right) b=b
$$

Replacing $t$ by $\left(1-a_{0} a\right) t$ and noting that $a\left(1-a_{0} a\right)=0$, we obtain successively $b\left(a_{0} a+\left(1-a_{0} a\right) t a\right)\left(a_{0}+\left(1-a_{0} a\right) t\right) b=b$ and $b\left(a_{0} a+\left(1-a_{0} a\right) t a\right)\left(a_{0}\right) b=b$ and so $b a_{0} b+b\left(1-a_{0} a\right) t a a_{0} b=b$. Since $b a_{0} b=b$ this gives $b\left(1-a_{0} a\right) t a a_{0} b=0$ for all $t \in R$. This leads to

$$
a a_{0} b\left(1-a_{0} a\right) t a a_{0} b\left(1-a_{0} a\right)=0 \quad \forall t \in R .
$$

The semiprimeness of $R$ implies that $a a_{0} b\left(1-a_{0} a\right)=0$. Left multiplying by $a_{0} \in \operatorname{ref}(a)$, we get that $a_{0} b\left(1-a_{0} a\right)=0$ and hence since $a_{0} \in I(b)$ we conclude that $b\left(1-a_{0} a\right)=0$. Therefore we obtain that $R b \subseteq R a$ and by symmetry $R a \subseteq R b$ and hence $R a=R b$. In the same way replacing $t$ by $t\left(1-a a_{0}\right)$ in (1), we obtain $a R=b R$. The Hartwig's Theorem then gives us that there exist invertible elements $u, v \in R$ such that $a=b u$ and $b=a v$. The argument at the end of the proof of the semiprime case (cf. Theorem 7 ) proves the theorem.

We now give an example of a ring, showing that without the semipriness hypothesis both of the above theorems are false.

Example 11. Consider the $\mathbb{F}_{2}$-algebra

$$
R=\mathbb{F}_{2}\left\langle a, b, x \mid a x a=a, b x b=b, x a x=x, x b x=x, a^{2}=b^{2}=a b=x^{2}=0\right\rangle
$$

This ring is finite and $\{a, b, x, a x, b x, x a, x b, a x b, b x a\}$ is a basis of $R$ as an $\mathbb{F}_{2 \text {-vector }}$ space. It is easy to determine that $r(a)=r(b)\langle a, b, a x, b x, a x b, b x a\rangle, l(a)=l(b)=$ $\langle a, b, x a, x b, a x b, b x a\rangle I(a)=x+R, I(b)=x+R, \operatorname{ref}(a)=\{x\}=\operatorname{ref}(b)$. Of course, $(R a R)^{2}=0$, showing that $R$ is not semiprime.

The next corollary is a direct consequence of Theorems 7 and 10 .

Corollary 12. Let $a, b$ be elements of a semiprime ring $R$. Then the following are equivalent:

(1) $I(a)=I(b)$,

(2) $a=b$,

(3) $\operatorname{ref}(a)=\operatorname{Ref}(b)$.

Remark 13. We close with the following comment. The question of the equality of two elements in a regular ring that have the same set of inner inverses arose while the authors have been working on the question: if, for a regular self-injective ring $R, I(c)=I(a)+I(b), a, b, c \in R$, is it true that $c$ is unique? If not, obtain a complete solution for c. We will discuss that in another paper which is in progress. 


\section{Acknowledgment}

We thank the referee for his/her valuable comments including the remark that our proof of Theorem 10 for prime rings can be carried to semiprime rings as well.

\section{REFERENCES}

[1] A. Ben-Israel and T.N.E. Greville, Generalized inverses: Theory and Applications, John Wiley and Sons New York (1974).

[2] S.K. Jain and K.M. Prasad, Right-left symmetry of $a R \oplus b R=(a+b) R$ in regular rings, Journal of Pure and Applied Algebra 133 (1998) 141-142.

[3] R.E. Hartwig, Generalized inverses, EP elements and associates, Revue Roumaine des Mathematiques Pures et Appliquees, (January 1978), 57-60.

Adel Alahmedi, Department of Mathematics, King abdulaziz University, Jeddah, SA, Email:adelnife2@yahoo.com; S. K. Jain, Department of Mathematics, King Andulaziz University Jeddah, SA,And, Ohio University, USA, Email:Jain@ohio.edu, Andre Leroy, Faculté Jean Perrin, Université D’Artois, Lens, France, Email:Andre.Leroy@univ-artois.fr 